\title{
Prostatic adenocarcinoma with a peculiar morphology - a rare case of pseudohyperplastic variant with inverted polarity
}

\author{
Daniel Abensur Athanazio ${ }^{1,2^{*}}$, Maiara Ferreira de Souza ${ }^{1}$ and Maria Estela Pompeu do Amaral ${ }^{1}$
}

\begin{abstract}
Background: The inverted (hobnail) variant of high-grade prostatic intraepithelial neoplasia (HGPIN) has been reported in two previous series and one case of inverted polarity in invasive adenocarcinoma has been reported. We reported an additional case of invasive carcinoma with this peculiar morphology.

Case presentation: We reported an additional case of invasive carcinoma with this peculiar morphology. A prostatectomy specimen of a 64-year-old patient showed a GG2 adenocarcinoma with extensive intraprostatic perineural infiltration and extraprostatic extension. Half of the entire tumor showed a distinctive inverted morphology.

Conclusion: Although pseudohyperplastic adenocarcinoma is believed to be a low-grade tumor to be graded as Gleason pattern 3, awareness of this morphology is important to collect more information on its biologic behavior and clinical implication.
\end{abstract}

Keywords: Prostate, Adenocarcinoma, Prostatic Intraepithelial Neoplasia

\section{Background}

The inverted (hobnail) variant of high-grade prostatic intraepithelial neoplasia (HGPIN) has been reported in two series (Argani and Epstein 2001; Öznur et al. 2015). Only one case of inverted polarity in invasive adenocarcinoma has been described (Öznur et al. 2015). We reported an additional case of invasive carcinoma with this peculiar morphology. Since these tumors are currently diagnosed as (low grade) pseudohyperplastic variant of prostatic adenocarcinoma, awareness of this morphology (or maybe sub-variant) is important to collect more data on the clinical implications of this distinctive morphology.

\footnotetext{
* Correspondence: dathanazio@gmail.com

'Imagepat, Laboratory of Pathology, Edf. Eventus Empresarial, 3० Andar Rua Lucaia, 209 Rio Vermelho, BA CEP 41940-660 Salvador, Brazil

${ }^{2}$ Hospital Universitário Professor Edgard Santos / Federal University of Bahia, Salvador, Bahia, Brazil
}

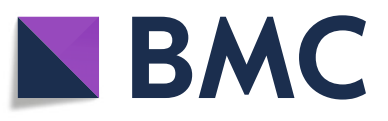

(๑) The Author(s). 2022 Open Access This article is licensed under a Creative Commons Attribution 4.0 International License, which permits use, sharing, adaptation, distribution and reproduction in any medium or format, as long as you give

appropriate credit to the original author(s) and the source, provide a link to the Creative Commons licence, and indicate if changes were made. The images or other third party material in this article are included in the article's Creative Commons licence, unless indicated otherwise in a credit line to the material. If material is not included in the article's Creative Commons licence and your intended use is not permitted by statutory regulation or exceeds the permitted use, you will need to obtain permission directly from the copyright holder. To view a copy of this licence, visit http://creativecommons.org/licenses/by/4.0/. 


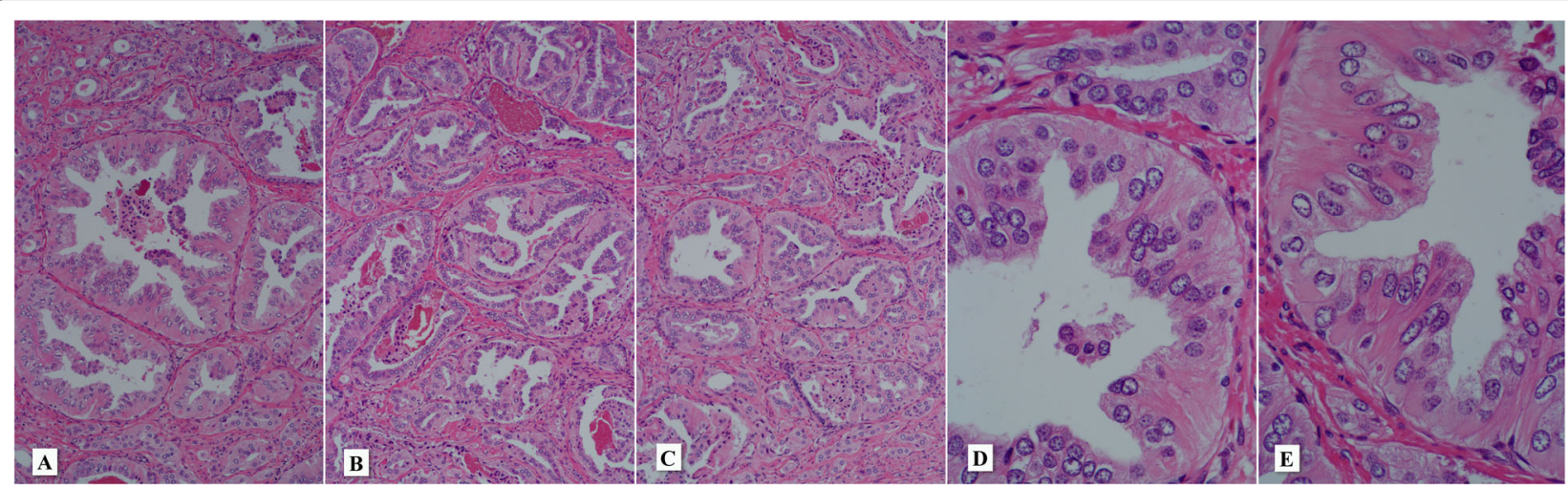

Fig. 1 Invasive prostatic adenocarcinoma with pseudohyperplastic morphology and inverse polarity. See nuclei arranged towards the lumen. Microscopic architecture is very reminiscent of high-grade prostatic neoplasia with inverted polarity. Hematoxylin and eosin stain: A,B and C (40x) and D and C (400x)

Despite the presence usual acinar adenocarcinoma of both Gleason pattern 3 and Gleason pattern 4 morphologies, $50 \%$ of the entire tumor was composed of a pseudohyperplastic component with peculiar, inverted polarity (Figs. 1 and 2). In these areas, almost all nuclei were arranged away from the basement membrane and located close to glandular lumina. This component showed extensive association with intraprostatic perineural infiltration and extraprostatic extension (Figs. 3 and 4). Immunohistochemical antibody cocktail staining (P63, high molecular weight cytokeratins and alphamethylacyl-CoA racemase, AMACR) showed that most of pseudohyperplastic / inverted component was devoid of basal cells and showed weak AMAC staining (Fig. 5). Minor areas with this morphology showed preserved basal cells which was consistent with the diagnosis of inverted HGPIN (see discussion).

\section{Discussion}

Twenty years ago, Argani and Epstein described the inverted (hobnail) variant of high-grade prostatic intraepithelial neoplasia (Argani and Epstein 2001). These lesions were characterized by the arrangement of nuclei polarized towards the lumen. Among 15 cases reported, seven were associated with invasive acinar adenocarcinoma (usual type). In 2015, Öznur and colleagues described additional 13 cases of inverted variant of HGPI $\mathrm{N}$, seven associated with invasive adenocarcinoma - and one of them with inverted polarity also in the invasive component (Öznur et al. 2015). To the best of our knowledge, this is the second reported case of pseudohyperplastic (HGPIN-like) invasive adenocarcinoma with inverted polarity.

In current practice, an invasive prostatic adenocarcinoma that resembles high-grade prostatic intraepithelial neoplasia is better designated pseudohyperplastic variant

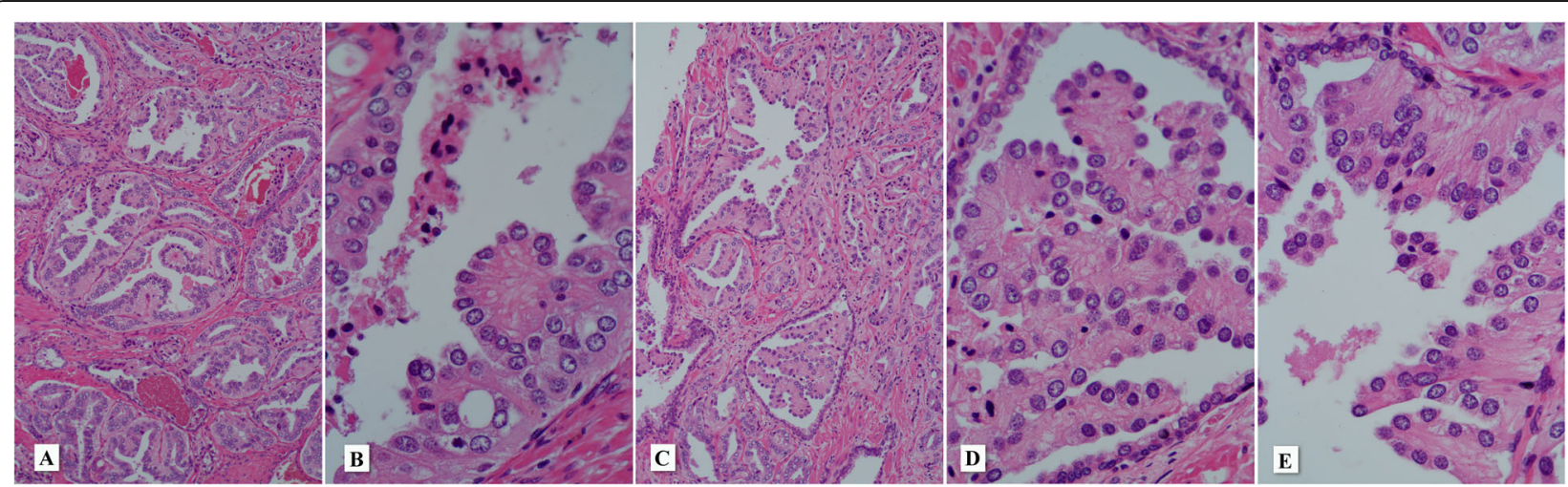

Fig. 2 Small micropapillary projections in invasive prostatic adenocarcinoma with pseudohyperplastic morphology and inverse polarity (A-D). This proliferation can be seen within preexistent ducts with bland double epithelial layer suggesting colonization of benign glands (C-E). Hematoxylin and eosin stain: A (100x), B (400x), C (100x), D (400x) and E (400x) 


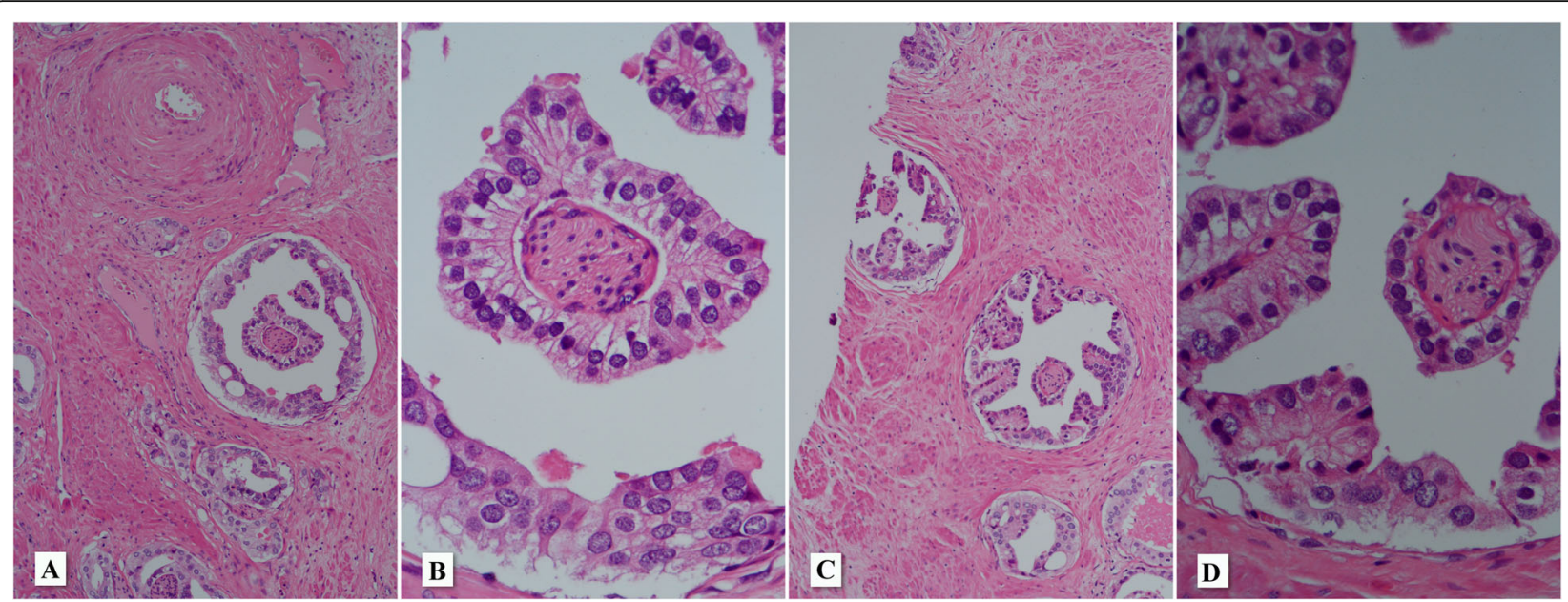

Fig. 3 Obvious perineural invasion by prostatic adenocarcinoma with pseudohyperplastic morphology and inverse polarity. Hematoxylin and eosin stain: A (100x), B (400x), C (100x) and D (400x)

(Paulk et al. 2018). Pseudohyperplastic variant shows dilated glands with papillary infolding which may be very reminiscent of benign glands. These glands area lined by a single layer of cells that show vesicular/round nuclei with macronucleoli or bland nuclei with deceptive benign appearance. Infiltrative pattern of growth, a high index of suspicious and awareness of this variant, and ancillary investigation of basal cells by immunohistochemistry are crucial for the diagnosis (Humphrey et al. 1998, Levi and Epstein 2000; Arista-Nasr et al. 2015; Humphrey 2018). These tumors are considered low-grade and graded as Gleason pattern 3 by consensus (Epstein et al. 2005). In contrast, ductal PIN-like adenocarcinoma is characterized by the same architectural changes but show pseudostratified lining. By definition, ductal adenocarcinoma of the prostate is composed by columnar/tall cells and graded as Gleason pattern 4. Recent data suggest that ductal adenocarcinomas with PIN-like architecture (PIN-like ductal adenocarcinoma) show a more indolent behavior and, therefore, should be also graded as Gleason pattern 3 (Paulk et al. 2018; Zhou 2018).

\section{Conclusions}

This is the second report case of invasive prostatic adenocarcinoma with pseudohyperplastic morphology

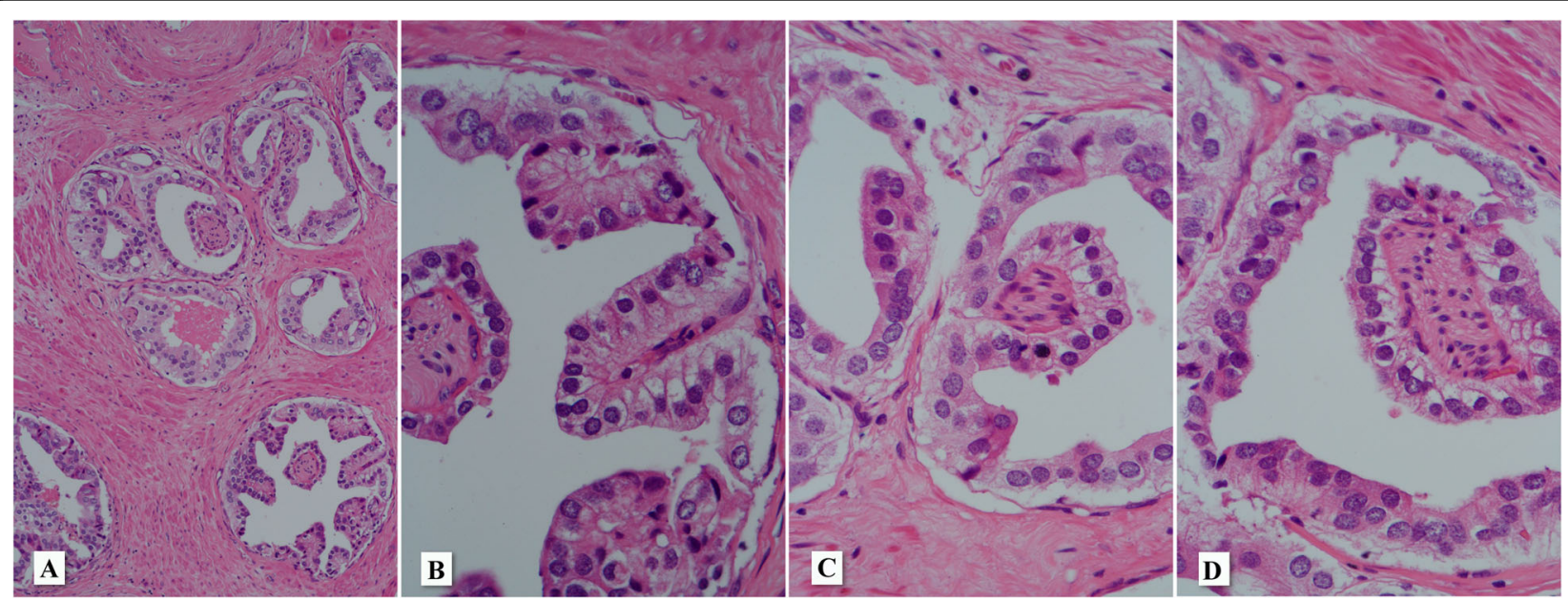

Fig. 4 Other illustrative areas of extensive perineural invasion by prostatic adenocarcinoma with pseudohyperplastic morphology and inverse polarity. Hematoxylin and eosin stain: A (100x), B (400x), C (100x) and D (400x) 


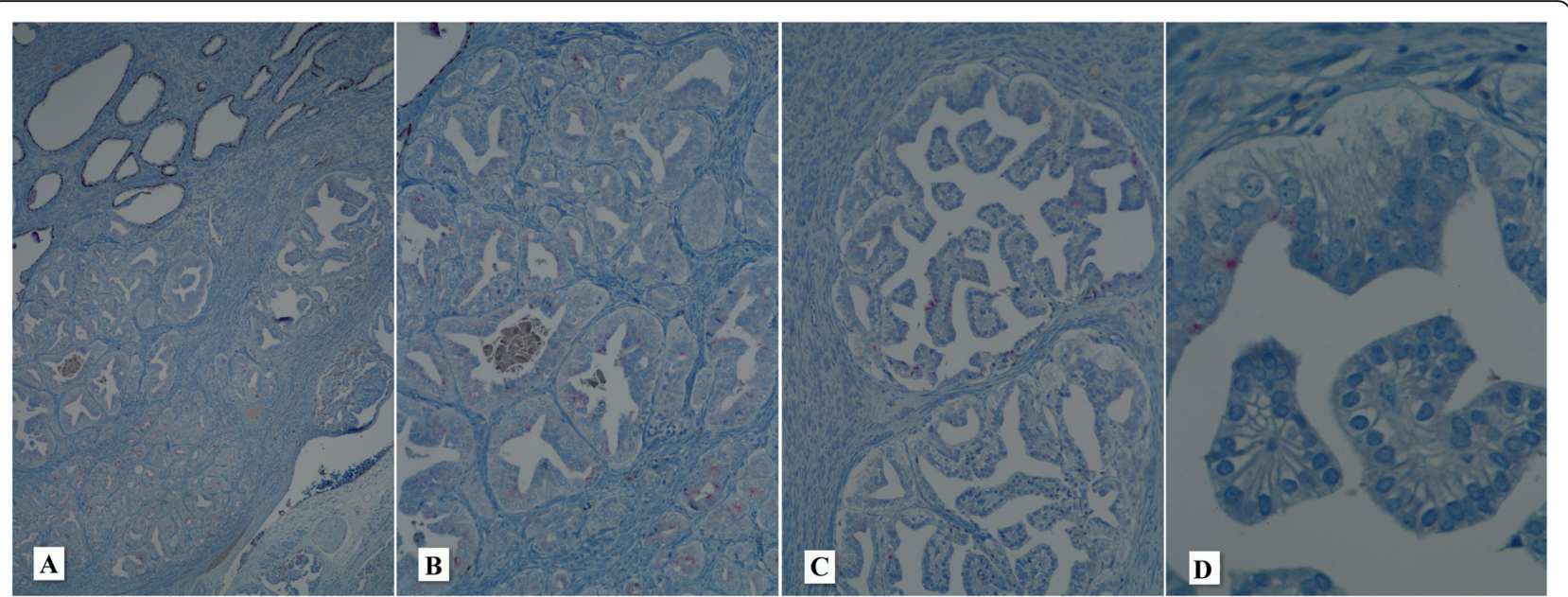

Fig. 5 Invasive prostatic adenocarcinoma with pseudohyperplastic morphology and inverse polarity showing absence of basal cells and weak cytoplasmic/luminal expression of alpha-methylacyl-CoA racemase. Immunohistochemical antibody cocktail staining - P63 and high molecular weight cytokeratins (peroxidase - brown) and alpha-methylacyl-CoA racemase (phosphatase - red): A (40x), B and C (100x) and D (400x)

and inverse polarity. Although pseudohyperplastic adenocarcinoma is believed to be a low-grade tumor to be graded as Gleason pattern 3, this particular tumor showed extensive extraprostatic extension and association with higher grade component. Awareness of this morphology is important to collect more information on its biologic behavior and clinical implication.

\section{Abbreviations}

AMACR: alpha-methylacyl-CoA racemase; HGPIN: Hematoxylin and eosin stain

\section{Acknowledgements}

None.

\section{Authors' contributions}

DAA conceived the idea. DAA was the major contributor to the writing of the manuscript. DDA, MFS and MEPA diagnosed the cases. MSF and MEPA were major contributors for critically revising the manuscript for important intellectual content. The authors read and approved the final manuscript.

\section{Funding}

This work has no funding sources.

\section{Availability of data and materials}

Supplementary data is available upon request.

\section{Declarations}

\section{Ethics approval and consent to participate}

Not applicable.

Written informed consent was obtained from the patient for participation in this case report. A copy of the written consent is available for review by the Editor-in-Chief of this journal.

\section{Consent for publication}

Written informed consent was obtained from the patient for publication of this case report and any accompanying images. A copy of the written consent is available for review by the Editor-in-Chief of this journal.

\section{Competing interests}

The authors declare that they have no competing interests.
Received: 15 November 2021 Accepted: 27 December 2021

Published online: 10 February 2022

\section{References}

Argani P, Epstein Jl. Inverted (Hobnail) high-grade prostatic intraepithelial neoplasia (PIN): Report of 15 cases of a previously undescribed pattern of high-grade PIN. Am J Surg Pathol. 2001;25:1534-9.

Arista-Nasr J, Martínez-Benítez B, Aguilar-Ayala EL, Aleman-Sanchez CN, BornsteinQuevedo L, Albores-Saavedra J. Pseudohyperplastic prostate carcinoma: histologic patterns and differential diagnosis. Ann Diagn Pathol. 2015;19(4): 253-60.

Epstein JI, Allsbrook WC Jr, Amin MB, Egevad LL. ISUP Grading Committee (2005) The 2005 International Society of Urological Pathology (ISUP) Consensus Conference on Gleason Grading of Prostatic Carcinoma. Am J Surg Pathol 29(9):1228-42.

Humphrey PA, Kaleem Z, Swanson PE, Vollmer RT. Pseudohyperplastic prostatic adenocarcinoma. Am J Surg Pathol. 1998;22(10):1239-46.

Humphrey PA. Variants of acinar adenocarcinoma of the prostate mimicking benign conditions. Mod Pathol. 2018;31(S1):64-70.

Levi AW, Epstein JI. Pseudohyperplastic prostatic adenocarcinoma on needle biopsy and simple prostatectomy. Am J Surg Pathol. 2000;4(8):1039-46.

Öznur M, Koca SB, Yildiz P, Bahadir B, Behzatoğlu K. Inverted (hobnail) high-grade prostatic intraepithelial neoplasia and invasive inverted pattern. Oncol Lett. 2015;10(4):2395-9.

Paulk A, Giannico G, Epstein JI. PIN-like (Ductal) Adenocarcinoma of the Prostate. Am J Surg Pathol. 2018;42(12):1693-700.

Zhou M. High-grade prostatic intraepithelial neoplasia, PIN-like carcinoma, ductal carcinoma, and intraductal carcinoma of the prostate. Mod Pathol. 2018; 31(S1):71-9.

\section{Publisher's Note}

Springer Nature remains neutral with regard to jurisdictional claims in published maps and institutional affiliations. 